\title{
5-Aminolevulinic acid-induced protoporphyrin IX with multi-dose ionizing irradiation enhances host antitumor response and strongly inhibits tumor growth in experimental glioma in vivo
}

\author{
JUNKOH YAMAMOTO $^{1}$, SHUN-ICHIRO OGURA ${ }^{2}$, SHOHEI SHIMAJIRI ${ }^{3}$, YOSHITERU NAKANO ${ }^{1}$, \\ DAISUKE AKIBA $^{1}$, TAKEHIRO KITAGAWA ${ }^{1}$, KUNIHIRO UETA ${ }^{1}$, TOHRU TANAKA ${ }^{4}$ and SHIGERU NISHIZAWA ${ }^{1}$ \\ ${ }^{1}$ Department of Neurosurgery, University of Occupational and Environmental Health, Kitakyushu, Fukuoka 807-8555; \\ ${ }^{2}$ Department of Bioengineering, Graduate School of Bioscience and Biotechnology, Tokyo Institute of Technology, Yokohama, \\ Kanagawa 226-8501; ${ }^{3}$ Department of Surgical Pathology, University of Occupational and Environmental Health, \\ Kitakyushu, Fukuoka 807-8555; ${ }^{4}$ SBI Pharmaceuticals Co., Ltd., Minato-ku, Tokyo 106-6020, Japan
}

Received January 10, 2014; Accepted November 3, 2014

DOI: $10.3892 / \mathrm{mmr} .2014 .2991$

\begin{abstract}
Ionizing irradiation is a well-established therapeutic modality for malignant gliomas. Due to its high cellular uptake, 5-aminolevulinic acid (ALA) is used for fluorescence-guided resection of malignant gliomas. We have previously shown that 5-ALA sensitizes glioma cells to irradiation in vitro. The aim of the present study was to assess whether 5-ALA acts as a radiosensitizer in experimental glioma in vivo. Rats were subcutaneously injected with $9 \mathrm{~L}$ gliosarcoma cells and administered 5-ALA. The accumulation of 5-ALA-induced protoporphyrin IX was confirmed by high-performance liquid chromatography (HPLC) analysis. Subcutaneous (s.c.) tumors were subsequently irradiated with 2 Gy/day for five consecutive days. In the experimental glioma model, high-performance liquid chromatography analysis revealed a high level of accumulation of 5-ALA-induced protoporphyrin IX in s.c. tumors $3 \mathrm{~h}$ after 5-ALA administration. Multi-dose ionizing irradiation induced greater inhibition of tumor growth in rats that were administered 5-ALA than in the non-5-ALA-treated animals. Immunohistochemical analysis of the s.c. tumors revealed that numerous ionized calcium-binding adapter molecule 1 (Iba1)-positive macrophages gathered at the surface of and within the s.c. tumors following multi-dose ionizing irradiation in combination with 5-ALA administration. By contrast, the s.c. tumors in the control group scarcely showed aggregation of Iba1-positive macrophages. These results suggested that multi-dose ionizing irradiation with 5-ALA induced not only a direct cytotoxic effect but also enhanced the host antitumor
\end{abstract}

Correspondence to: Dr Junkoh Yamamoto, Department of Neurosurgery, University of Occupational and Environmental Health, 1-1 Iseigaoka, Yahatanishi-ku, Kitakyushu, Fukuoka 807-8556, Japan E-mail: yama9218@med.uoeh-u.ac.jp

Key words: glioblastoma, radiosensitizer, macrophage, ionized calcium-binding adapter molecule 1 , microdensitometry, phagocytosis immune response and thus caused high inhibition of tumor growth in experimental glioma.

\section{Introduction}

Photodynamic therapy (PDT) with photosensitizers, including hematoporphyrin derivatives $(\mathrm{HpD})$, photofrin and 5-aminolevulinic acid (ALA)-induced protoporphyrin IX (PpIX), has been used in the treatment of several types of cancer (1-3). The effectiveness of photosensitizers depends on two important properties: The production of cytotoxic oxygen and fluorescence upon excitation by laser irradiation (4-6). In particular, 5-ALA has high affinity for malignant gliomas, and thus, it is commonly used for fluorescence-guided tumor resection in clinical neurosurgery (4). Although several porphyrin compounds, including HpD and photofrin, have been shown to act as radiosensitizers, the radiosensitizing activity of 5-ALA-induced PpIX remains controversial (7-11). We previously demonstrated that although the ability of 5-ALA-induced PpIX to radiosensitize glioma cell lines in vitro was weak, multi-dose ionizing irradiation may be used to enhance this radiosensitizing effect (12). The aim of the present study was to further evaluate the radiosensitizing effects of 5-ALA-induced PpIX in combination with multi-dose ionizing irradiation by assaying this activity in a rat subcutaneous (s.c.) glioma model. The potency of 5-ALA-induced PpIX as a radiosensitizer for cancer therapy was also discussed.

\section{Materials and methods}

Chemicals. 5-ALA was purchased from Cosmo Bio K.K. (Tokyo, Japan) and was dissolved in phosphate-buffered saline (PBS; WAKO Pure Chemical Industries, Ltd, Osaka, Japan) at a concentration of $100 \mathrm{mg} / \mathrm{ml}$. The $\mathrm{pH}$ of the solution was adjusted to 6.0-6.3 with $10 \mathrm{~N}$ sodium hydroxide $(\mathrm{NaOH}$; WAKO Pure Chemical Industries, Ltd) and checked using $\mathrm{pH}$ indicator paper. The solution was used within $10 \mathrm{~min}$ of preparation. 5-ALA was intravenously administered to rats via the tail vein at a dose of $100 \mathrm{mg} / \mathrm{kg}$ body weight. 
Brain tumor cell lines and animals. All the following experiments were performed in accordance with an animal protocol approved by the Institutional Animal Care and Use Committee (University of Occupational and Environmental Health, Kitakyushu, Japan). The 9L gliosarcoma cell line, which was generated from inbred Fischer rats, has been widely used as a syngeneic rat model for experimental gliomas. Originally produced by $N$-methyl-nitrosourea mutagenesis in Fischer rats by Benda et al (13) and Schmidek et al (14) at Massachusetts General Hospital, the tumor was obtained by Barker at the University of California, cloned and designated 9L gliosarcoma due to the dual appearance of a glioblastoma and a sarcoma. 9L gliosarcoma cells rapidly proliferate under in vitro and in vivo conditions, and are the most widely used cells in experimental rat glioma, for example in brain and subcutaneous tumor models $(6,15)$. 9L gliosarcoma cells were obtained from Dr Tsutomu Tokuyama at the Hamamatsu University School of Medicine (Hamamatsu, Japan) and cultured for several days in RPMI-1640 medium (WAKO Pure Chemical Industries, Ltd) supplemented with $10 \%$ fetal calf serum (FCS; Nichirei Biosciences Inc., Tokyo, Japan) before use. Syngeneic male Fischer 344 rats ( 8 weeks of age; mean body weight, $167 \mathrm{~g}$ ) were purchased from SLC, Inc. (Hamamatsu, Japan). A total of 32 rats were used for the present study. Animals were inoculated with 9L gliosarcoma cells, as previously described $(3,6)$. Briefly, these cells $\left(1 \times 10^{6}\right)$ were implanted into the dorsal skin of the Fisher 344 rats, and thereby, a rat s.c. tumor model was prepared for the following experiments. All animals were kept at a constant room temperature of $24^{\circ} \mathrm{C}$ under a 12-h light/dark cycle ( 7 am to $7 \mathrm{pm}$ ) in the laboratory animal center of the University of Occupational and Environmental Health. In addition, all animals received sufficient food, which was sterilized and certified for experimental animals (MF; Oriental Yeast Co., Ltd, Tokyo, Japan), according to the animals requirements.

Evaluation of the accumulation of 5-ALA-induced PpIX in the rat s.c.tumor model. Firstly, the accumulation of 5-ALA-induced PpIX was confirmed in the established rat s.c. tumor model using high-performance liquid chromatography (HPLC) analysis and fluorescence observation. Once the tumors had grown to $\sim 1 \mathrm{~cm}$ in diameter, the rats were given an intravenous (i.v.) injection of 5-ALA (100 mg/kg body weight) into the tail vein. Three hours later, the rats were anesthetized and tumor specimens without the dorsal skin cover were removed and immediately snap-frozen in liquid nitrogen, then stored at $-80^{\circ} \mathrm{C}$ in the dark for HPLC analysis. According to the previously described method of HPLC analysis of porphyrin metabolites $(16,17)$, tumor specimens (1-mm diameter) were treated with $200 \mu \mathrm{l}$ of $0.1 \mathrm{M} \mathrm{NaOH}$ and homogenized on ice with a Powermasher II (Assist, Tokyo, Japan). An aliquot (10 $\mu \mathrm{l})$ of the NaOH-treated samples was withdrawn and used for a protein concentration assay (Quick Start ${ }^{\mathrm{TM}}$ Bradford Dye Reagent, Bio-Rad Laboratories, Inc., Hercules, CA, USA), while the remaining $50 \mu \mathrm{l}$ of cellular proteins were denatured by the addition of $150 \mu 1 N, N$-dimethylformamide/isopropanol solution (100:1, v/v; Nacalai Tesque, Inc., Kyoto, Japan). After overnight storage in the dark, the prepared samples were subjected to HPLC analysis performed as previously described $(2,17)$ with certain modifications. Briefly, porphyrins were separated using a Prominance HPLC system (Shimadzu, Kyoto, Japan) equipped with a reversed-phase C18 column (CAPCELL PAK, C18, SG300, $5 \mu \mathrm{m}, 4.6 \mathrm{~mm} \times 250 \mathrm{~mm}$; Shiseido, Tokyo, Japan) maintained at $40^{\circ} \mathrm{C}$. The elution solvents used were solvent A (1 M ammonium acetate including $12.5 \%$ acetonitrile, $\mathrm{pH} 5.2)$ and solvent $\mathrm{B}$ (50 $\mathrm{mm}$ ammonium acetate including $80 \%$ acetonitrile, $\mathrm{pH} 5.2$; Kanto Chemical Co., Inc., Tokyo, Japan). Elution was performed with solvent A for $5 \mathrm{~min}$ and subsequently with a linear gradient of solvent B (0-100\%) for $25 \mathrm{~min}$, followed by elution with solvent $\mathrm{B}$ for $10 \mathrm{~min}$. The elution flow was maintained at a constant rate through the use of a fluorospectrometer (excitation at a wavelength of $404 \mathrm{~nm}$, detection at a wavelength of $624 \mathrm{~nm}$; F7000; Hitachi, Tokyo, Japan). The porphyrin concentrations in the samples were estimated using calibration curves obtained with standard porphyrins (Protoporphyrin IX; Sigma-Aldrich, St. Louis, MO, USA).

In addition, under anesthesia, the rat dorsal skin covering the inoculated tumors was evaginated and the s.c. tumors were observed underneath the skin at $3 \mathrm{~h}$ post-intravenous administration of $100 \mathrm{mg} / \mathrm{kg}$ 5-ALA. Bright-field images of the s.c. tumors were captured using a digital camera (D90, Nikon Corporation, Tokyo, Japan) with a long-pass filter and an external halogen lamp as the light source (C-FID, Nikon). Subsequently, the same s.c. tumors were illuminated with ultraviolet light (410-nm light-emitting diode illuminator, SBI Pharma CO., Ltd., Tokyo, Japan), and tumor images were captured using a digital camera with a long-pass filter.

Evaluation of the in vivo radiosensitizing effects of 5-ALA with multi-dose ionizing irradiation in a rat s.c. tumor model. Syngeneic Fischer 344 rats were inoculated with 9L gliosarcoma cells, as previously described $(3,6)$. A previous study demonstrated that the growth of s.c. 9L tumors in syngeneic Fischer rats was inhibited at $>10$ Gy with single-dose ionizing irradiation (18). Other previous studies reported that for PDT, the dose of 5-ALA used for a single i.v. administration was $100-500 \mathrm{mg} / \mathrm{kg}$ for rodents $(6,19-21)$. Thus, in the present study, the maximal dose of ionizing irradiation and 5-ALA administration used were $10 \mathrm{~Gy}$ and $500 \mathrm{mg} / \mathrm{kg}$. A previous study by our group confirmed that multi-dose ionizing irradiation with 5-ALA inhibited tumor proliferation in vitro (12). However, multiple i.v. injection via the rat tail vein was technically difficult due to injury and obstruction of vessels. According to preliminary experiments by our group, up to 5 i.v. injections were possible. Therefore, the optimal ionizing irradiation schedule was determined to be 2 Gy and 5-ALA administration $(100 \mathrm{mg} / \mathrm{kg})$ per day, for five consecutive days. Once the s.c. tumors had grown to a diameter of 6-8 $\mathrm{mm}$, the animals were randomly divided into four groups and treated as follows: Control group, no further treatment $(n=5)$; 5-ALA administration $(n=5)$; ionizing irradiation $(\mathrm{RT})(\mathrm{n}=7)$; and ionizing irradiation with 5-ALA administration (5-ALA + RT) (n=7). In the 5-ALA administration group, the animals received 5-ALA (100 mg/kg) alone for five consecutive days without ionizing irradiation. In the ionizing irradiation with 5-ALA administration group, the animals were anesthetized $3 \mathrm{~h}$ post-5-ALA administration and the s.c. tumors were irradiated with 2 Gy in the dark using an X-ray irradiator (MBR-1520R; HITACHI Engineering \& Service Co., Ltd., Japan) at a rate of $0.65 \mathrm{~Gy} / \mathrm{min}$. The animals were completely covered with an X-ray shield sheet, except for the tumor regions, to avoid excessive exposure 

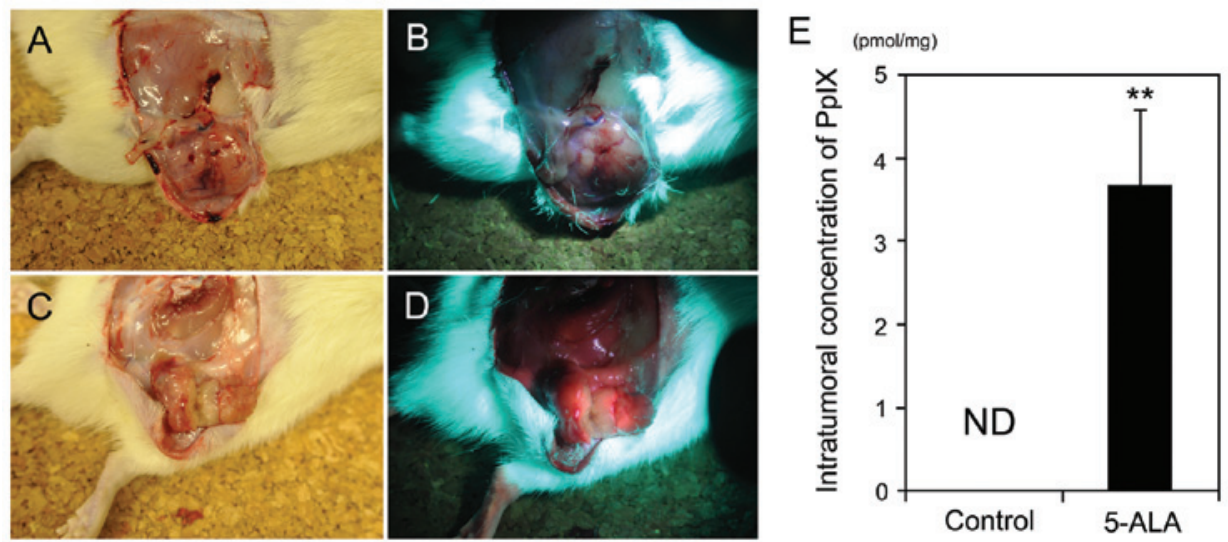

Figure 1. Representative images of a rat s.c. tumors following surgical exposure and HPLC analysis of the intratumoral concentration of PpIX (A and C) Bright-field image showing the rat s.c. tumor. (B and D) Fluorescence image showing the tumor illuminated by light of wavelength $410 \mathrm{~nm}$. (D) The s.c. tumor strongly expressed red fluorescence, originating from porphyrin, $3 \mathrm{~h}$ after the intravenous administration of 5-ALA. as compared with (B) the control tumor. (E) HPLC analysis showed a high accumulation of 5-ALA-induced PpIX in the tumor after 5-ALA administration (E). Data are presented as the mean (pmol/mg-protein) \pm the standard error of the mean. ${ }^{* *} \mathrm{P}<0.01$ compared with the control; ND, not detected. 5-ALA, 5-aminolevulinic acid; PpIX, protoporphyrin IX. HPLC, high-performance liquid chromatography; s.c., subcutaneous.

of the rest of the body to the ionizing irradiation. This procedure was performed for five consecutive days, resulting in exposure to a total of 10 Gy (2 Gy/day, 5 days). In the ionizing irradiation group, the s.c. tumors were irradiated in an identical manner, without the administration of 5-ALA. To avoid photochemical effects, all animals were kept in the dark for $12 \mathrm{~h}$ after 5-ALA administration. Thereafter, direct exposure of the animals to light was avoided. Tumor growth was assessed every 2 days until 16 days post-treatment. Tumor volumes were calculated using the formula $\mathrm{V}=\mathrm{a}^{2} \mathrm{~b} / 2$, where $\mathrm{a}$ and $\mathrm{b}$ are the shortest and longest perpendicular diameters, respectively (22). Sixteen days after treatment, the animals were sacrificed under deep anesthesia using equithesin $(0.4 \mathrm{ml} / 100 \mathrm{~g})$, which was composed of chloral hydrate, magnesium sulfate, ethanol and propylene glycol, all purchased from WAKO Pure Chemical Industries Ltd, as well as pentobarbital sodium salt (Tokyo Chemical Industry Co., Ltd, Tokyo, Japan). All tumor specimens were immediately removed with the dorsal skin cover and fixed in $20 \%$ formaldehyde/PBS for further pathological examination.

Pathological examination. Following fixation all tumor specimens were cut at the center of the tumor in the direction of the longest axis. Sections were stained with hematoxylin and eosin and ionized calcium-binding adapter molecule 1 (Iba1) for macrophage detection, and all staining processes were outsourced to Pathology Institute Corp, Toyama, Japan. Briefly, after a water bath pretreatment $\left(40 \mathrm{~min}, 95^{\circ} \mathrm{C}\right)$, deparaffinized sections were washed with KN buffer (KN-09002; Pathology Institute Corp., Toyama, Japan). The sections were incubated with goat polyclonal anti-Iba1 (1:3,000, ab107159; Abcam, Cambridge, UK) for $30 \mathrm{~min}$, followed by a further wash with KN buffer. Subsequently, the sections were incubated with Simple Stain ${ }^{\mathrm{TM}}$ MAX-PO (G) (H1301; Nichirei Bioscience Inc.) for $30 \mathrm{~min}$. Following a final wash with KN buffer, color development was performed with diaminobenzidine (DAB; DAKO, Glostrup, Denmark) for $10 \mathrm{~min}$ and the sections were counterstained with hematoxylin (WAKO Pure Chemical industries, Ltd). Thereafter, all tumor specimens were evaluated in our laboratory. Based on a previous study (23), microdensitometry for quantitative evaluation of Ibal immunohistochemistry was performed on digital microphotographs (Colorio EP-705A; Seiko Epson Corp., Suwa, Japan) using the public domain software Image J 1.46r (National Institutes of Health, Bethesda, MD, USA), with certain modifications of the original method. In brief, all Iba1-stained tumor specimens were scanned using a color scanner (Colorio EP-705A) and then photographed. To perform quantification of the immunohistochemical DAB color signal of Iba1, image data of all samples were transferred to Image J and converted to an 8-bit grey-scale image. The freehand tool was used to delineate the whole-tumor section as the region of interest (ROI) for in each tumor specimen, and the mean gray value (MGV) within the ROI was plotted on a graph. The representative value was defined as the average MGV of all the five tumor specimens in the control group and the relative intensity of the MGV of the other groups was calculated according to the representative value.

Statistical analyses. Data are presented as the mean \pm the standard error of the mean. Statistical analyses were performed using StatView 5.0 software (SAS Institute, Cary, NC, USA) The mean tumor volume was analyzed using unpaired two-sample $t$-tests, and the relative intensity of MGV of Ibal was calculated using Fisher's exact probability tests. Statistical significance was defined as $\mathrm{P}<0.05$.

\section{Results}

Intratumoral accumulation of 5-ALA-induced PpIX in the rat s.c. tumor model. In the established rat s.c. tumor model, tumors demonstrated strong fluorescence $3 \mathrm{~h}$ after the intravenous administration of 5-ALA, compared with that of the control tumors without 5-ALA administration (Fig. 1). Furthermore, HPLC analysis revealed that the amount of 5-ALA-induced PpIX in tumors was $3.66 \pm 0.91 \mathrm{pmol} / \mathrm{mg}$-protein $3 \mathrm{~h}$ after the intravenous administration of 5-ALA, which was significantly higher than that of the control $(\mathrm{P}<0.01)$ (Fig. 1E). By contrast, 5-ALA-induced PpIX was not detected in the control tumors without 5-ALA administration ( $<0.1 \mathrm{pmol} / \mathrm{mg}$-protein). 
Table I. Effect of 5-ALA and multi-dose RT on tumor growth.

\begin{tabular}{lcccccc}
\hline Group & Day 0 & Day 8 & Day 10 & Day 12 & Day 14 & Day 16 \\
\hline Control (n=5) & $142.3 \pm 13.8$ & $1191.5 \pm 128.8$ & $1793.2 \pm 199.2$ & $2296.8 \pm 233.3$ & $2799.0 \pm 281.9$ & $3747.1 \pm 254.1$ \\
ALA (n=5) & $143.9 \pm 17.4$ & $865.4 \pm 107.2$ & $1403.0 \pm 224.3$ & $1842.6 \pm 218.8$ & $2276.3 \pm 192.8$ & $2869.4 \pm 267.9$ \\
RT (n=7) & $149.9 \pm 14.9$ & $683.3 \pm 99.4$ & $1059.0 \pm 101.7$ & $1485.8 \pm 133.4$ & $1736.9 \pm 162.9$ & $2035.2 \pm 245.9$ \\
ALA + RT (n=7) & $146.0 \pm 12.9$ & $385.3 \pm 59.7$ & $495.6 \pm 91.1$ & $667.6 \pm 119.7$ & $819.0 \pm 135.5$ & $863.1 \pm 147.4$ \\
P-value $^{\mathrm{a}}$ & 0.8457 & 0.0246 & 0.0014 & 0.0007 & 0.001 & 0.0015 \\
\hline
\end{tabular}

Values are expressed as the mean tumor volume $\left(\mathrm{mm}^{3}\right) \pm$ the standard error of the mean. Brackets indicate number of sample. ${ }^{\mathrm{a}} \mathrm{RT}$ vs ALA $+\mathrm{RT}$ (unpaired t-test). 5-ALA, 5-aminolevulinic acid; RT, ionizing irradiation.
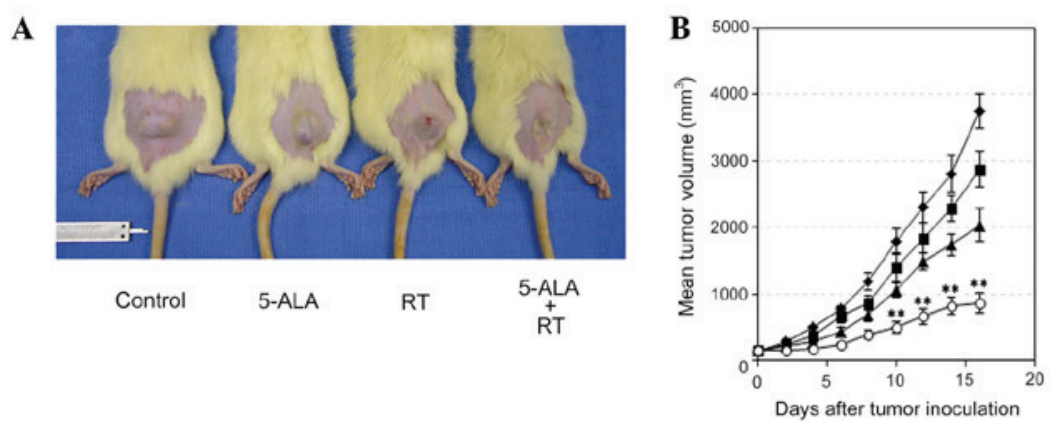

Figure 2. Representative image of rat subcutaneous (s.c.) tumors at day 16 after treatment. (A) The hair covering the skin was removed. (B) Growth curves of the s.c. tumors. Once the s.c. tumors had grown to a diameter of $6-8 \mathrm{~mm}$, the animals were randomly divided into four groups and treated as follows: ( $\downarrow$ ) Control $(\mathrm{n}=5) ;(\boldsymbol{\square})$ 5-ALA (n=5); ( $\mathbf{\Delta})$ multi-dose RT (n=7); and (o) multi-dose RT with 5-ALA (n=7). The control group did not receive any treatment. Rats in the 5-ALA group received 5-ALA $(100 \mathrm{mg} / \mathrm{kg})$ via the tail vein for five consecutive days. Rats in the multi-dose ionizing irradiation group were exposed to 2 Gy/day of RT for five consecutive days (total $10 \mathrm{~Gy}$ ). Rats in the multi-dose irradiation with 5-ALA group were exposed to RT (2 Gy/day) 3 h after the intravenous administration of 5-ALA $(100 \mathrm{mg} / \mathrm{kg})$ for five consecutive days. Each point represents the mean, and the bars represent the standard error of the mean; ${ }^{* *} \mathrm{P}<0.01$ versus the multi-dose RT group (A). s.c., subcutaneous; 5-ALA, 5-aminolevulinic acid; RT, ionizing irradiation.

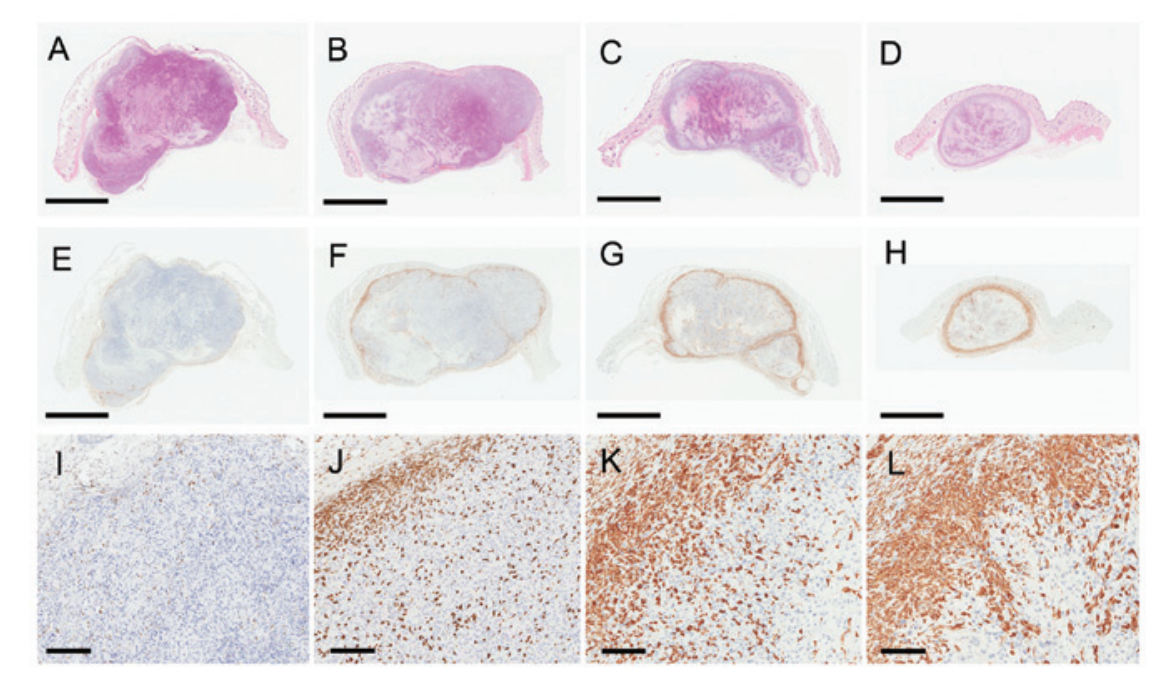

Figure 3. Pathology results of sc tumors at day 16 after treatment in the (A, E, I) control, (B, F, J) 5-ALA, (C, G, K) multi-dose RT, and (D, H, L) multi-dose RT with 5-ALA groups. (A-D) (HE) staining and (E-L) Iba1 staining. (E-H) The Iba1-stained sections correspond to the HE-stained sections in each group. (I-L) Magnified views of the Iba1 staining of the surface of the s.c. tumors in each group. (A-D) The s.c. tumors showed coagulative necrosis in each group. (H, L) Iba1-positive macrophages gathered at the surface of, and within, the s.c. tumors in the multi-dose ionizing irradiation with 5-ALA group. In contrast, (E, I) Iba1-positive macrophages were scarcely observed in the control group. Scale bar: (A-H) $5 \mathrm{~mm}$ and (I-L) $200 \mu \mathrm{m}$. 5-ALA, 5-aminolevulinic acid; RT, ionizing irradiation; HE, hematoxylin and eosin; Iba1, ionized calcium-binding adapter molecule 1.

Radiosensitizing effect of 5-ALA with multi-dose ionizing irradiation in vivo. The subcutaneously inoculated 9L gliosarcomas grew at a near exponential rate (Fig. 2, Table I). On the first day (day 0) of tumor treatment, there was no difference in tumor size between the RT and 5-ALA + RT groups $(\mathrm{P}=0.8457$, Table I). Treatment with multi-dose ionizing irradiation and 5-ALA 

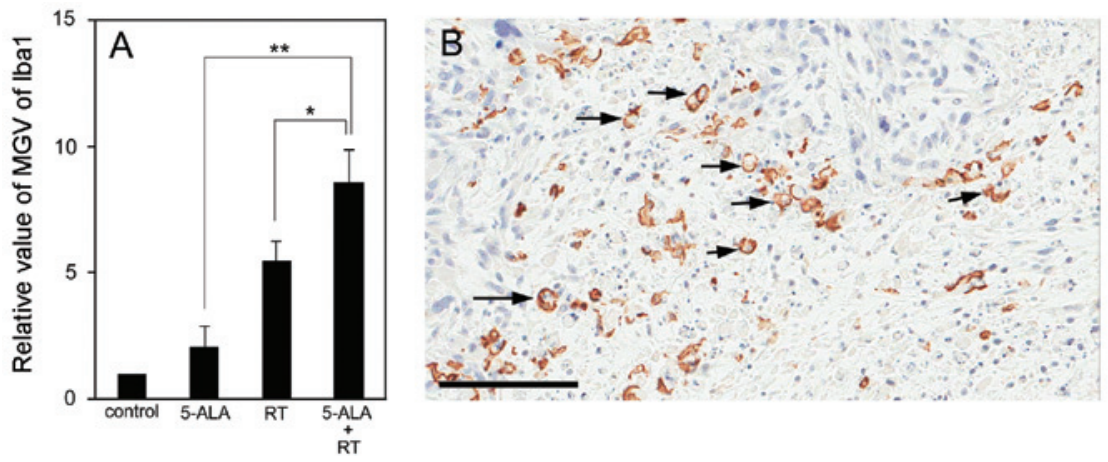

Figure 4. Microdensitometric analysis. (A) Relative intensity of the MGVs of Iba1. The MGV of the multi-dose ionizing irradiation with 5-ALA group was significantly higher than that of the other groups. (B) Representative pathological findings of the boundary zone between the coagulation necrosis and the surviving tumor cells in the subcutaneous tumor in the multi-dose ionizing irradiation with 5-ALA group (Iba1 staining). Iba1-positive macrophages gathered in the boundary zone between the coagulation necrosis and the surviving tumor cells. Note that numerous Iba1-positive macrophages showed phagocytic features (arrows). Values are expressed as the mean \pm the standard error of the mean. ${ }^{*} \mathrm{P}<0.05$ and ${ }^{* *} \mathrm{P}<0.01$ compared with the control (A). Scale bar: $120 \mu \mathrm{m}$. MGV, mean gray value; 5-ALA, 5-aminolevulinic acid; RT, ionizing irradiation; Iba1, ionized calcium-binding adapter molecule 1.

significantly inhibited tumor growth, compared with that of tumors treated with irradiation alone $(\mathrm{P}<0.01$, day 10) (Fig. 2 and Table I). On day 16 , the mean inhibition of tumor growth in the 5-ALA + RT group was $42.5 \%$ of that in the RT group (Fig. $2 \mathrm{~A}$ and Table I). OF note, tumor growth was inhibited by 5 -ALA administration without treatment with ionizing irradiation as compared with that of the control group ( $\mathrm{P}=0.0448$ at day 16$)$.

Histological evaluation of s.c.9L gliosarcomas after multi-dose ionizing irradiation. The tumor specimens showed coagulative necrosis within the tumors, with variance among the groups (Fig. 3A-D). Of note, the Iba1-positive macrophages also showed a certain variance among the groups (Fig. 3E-L). The control group showed a very low accumulation of Iba1-positive macrophages in the s.c. tumors (Fig. 3E and I). In the 5-ALA group, Iba1-positive macrophages mainly gathered at the surface of the s.c. tumors, only slightly invading the tumors (Fig. 3E and J). Similarly, numerous Iba1-positive macrophages gathered at the surface of and within the s.c. tumors following treatment with ionizing irradiation (Fig. 3G and $\mathrm{K}$ ) and in particular following treatment with ionizing irradiation in combination with 5-ALA (Fig. 3H and L). Microdensitometric analysis showed that significantly more Iba1-positive macrophages gathered in the s.c. tumors of the ionizing irradiation + 5-ALA group compared with those of the other groups $(\mathrm{P}<0.05$ vs. RT; $\mathrm{P}<0.01$ vs. 5-ALA) (Fig. 4A). Analysis of the distribution of the Iba1-positive macrophages within the tumors revealed that the Iba1-positive macrophages did not gather within the zone of coagulation necrosis, but primarily gathered in the boundary zones between the coagulation necrosis and the surviving tumor cells. In particular, a number of Iba1-positive macrophages displayed features of the phagocytic process (Fig. 4B).

\section{Discussion}

The results of the present study confirmed that 5-ALA-induced PpIX has a radiosensitizing effect in experimental glioma. It was determined that tumor growth was significantly inhibited in 5-ALA-treated irradiated rats compared with rats exposed to multi-dose ionizing irradiation in the absence of 5-ALA. This was in agreement with a previous in vitro study by our group (12). To the best of our knowledge, the present study was the first to show the radiosensitizing effects of 5-ALA-induced PpIX and to confirm the immunological effects in experimental glioma in vivo.

The mechanism underlying the radiosensitizing effects of porphyrin compounds remains elusive. Using confocal laser scanning microscopy, it was previously demonstrated that intracellular 5-ALA-induced PpIX has an important role in the production of reactive oxygen species and in radiosensitization (12). This radiosensitizing effect was found to depend on the intracellular concentrations of porphyrin compounds, including $\mathrm{HpD}$ and photofrin, which were low following 5-ALA administration (6). Therefore, high intracellular concentrations of $\mathrm{HpD}$ and photofrin may have a strong radiosensitizing effect with single-dose ionizing irradiation, comparable with that of 5-ALA-induced PpIX in vitro and in vivo (8-10, 24). In certain cell lines, however, 5-ALA-induced PpIX was shown to have a low sensitizing effect with single-dose ionizing irradiation $(12,25)$. In addition, another study indicated that ionizing irradiation increased, rather than inhibited, ALA-induced synthesis of PpIX in human colon adenocarcinoma cells in vitro (9). In the present study, 5-ALA-induced PpIX was found to significantly enhance tumor sensitivity to multi-dose ionizing irradiation. Therefore, repeated administration of 5-ALA, combined with ionizing irradiation, may enhance the radiosensitizing effect of 5-ALA-induced PpIX, thereby strongly inhibiting tumor growth in experimental glioma.

In addition, the immunological response to 5-ALA-induced PpIX and multi-dose ionizing irradiation was measured by performing immunohistochemical staining with Iba1 for macrophages. The combination of 5-ALA-induced PpIX with multi-dose ionizing irradiation resulted in a strong aggregation of Iba1-positive macrophages at the surface of and within the s.c. tumors. Iba1 expression is typically upregulated in activated macrophages/microglia, which exhibit a distinct morphology with an amoeboid shape and short processes $(26,27)$. Macrophages may be broadly divided into the following two groups: $i$ ) Classically activated M1-type macrophages that typically participate in the coordinated response to immunogenic antigens, primarily through the production of proinflammatory mediators, including interleukin (IL)-1B, IL-12 and tumor necrosis factor- $\alpha$ (TNF- $\alpha$ ), and have an overall enhanced ability to phagocytose 
pathogenic material $(28,29)$; and ii) alternatively activated M2-type macrophages that do not secrete proinflammatory mediators such as IL-1B and TNF- $\alpha$ (30) and are believed to exert immunomodulation primarily through the secretion of the potent immunosuppressive cytokines IL-10 and TGF- $\beta$ and have a decreased phagocytic capacity $(31,32)$. In the present study, numerous Iba1-positive macrophages gathered at the surface of and within the s.c. tumors following 5-ALA administration of multi-dose ionizing irradiation. In particular, these Iba1-positive macrophages with phagocytic features primarily gathered in the boundary zones between the coagulation necrosis and the surviving tumor cells. By contrast, although coagulative necrotic changes were revealed within the s.c. tumors in all the groups, Iba1-positive macrophages scarcely gathered at the surface of the s.c. tumors in the control group. Thus, these Iba1-positive macrophages did not gather at the surface of the s.c. tumors merely for the removal of the coagulative necrotic tissues within the tumors. Therefore, it is hypothesized that 5-ALA-induced PpIX with multi-dose ionizing irradiation treatment induced not only a direct cytotoxic effect, but additionally a long-lasting immunological effect following the ionizing irradiation, including the induction of tumor cytotoxic M1-type macrophages, and consequently caused a strong inhibition of tumor growth.

A previous study reported that photodynamic therapy with 5-ALA led to an increase in tumor-infiltrating mononuclear cells and the activation of intraperitoneal macrophages in Lewis lung carcinoma (33). Of note, the present study found that repeated administration of 5-ALA alone inhibited tumor growth. While it was not possible to completely exclude all photodynamic effects, the results indicated that even when exposure to room light was limited and indirect, 5-ALA alone enhanced the host antitumor immune response. It has been suggested that the mechanisms of these immunological effects with response to 5-ALA in cancer therapy shall be investigated in greater detail in future studies (34). A recent clinical study showed that 5-ALA administered orally once a day over a 12 -week period reduced both fasting and postprandial glucose levels in type-2 diabetes mellitus without adverse effects (35). Patients with malignant brain tumors frequently receive fractionated radiotherapy following surgical resection. Various radiotherapy modalities, including stereotactic radiotherapy (SRT), stereotactic radiosurgery (SRS) and intensity-modulated radiotherapy, precisely control the intensity of ionizing irradiation, thereby avoiding or reducing the exposure of healthy tissues and limiting the side-effects of the treatment. Thus, SRT and SRS provide high doses of irradiation to small, precise areas of intracranial lesions $(36,37)$. 5-ALA has a high affinity for malignant brain tumors and has a number of advantages, including reduced skin phototoxicity compared with that to other photosensitizers. The present study presents preliminary data of the radiosensitizing effects of 5-ALA-induced PpIX in experimental glioma. Multi-dose ionizing irradiation with 5-ALA-induced PpIX may be a novel therapeutic strategy for malignant gliomas with possible clinical applications.

The present study demonstrates the radiosensitizing effect of 5-ALA in experimental glioma in vivo. Multi-dose ionizing irradiation alongside 5-ALA administration induces strong inhibition of tumor growth and enhances the antitumor immune response in experimental glioma. Although investi- gation of the mechanisms underlying the combined effects of multi-dose irradiation and 5-ALA-induced PpIX is essential to clearly define the interactions between these components, multi-dose ionizing irradiation with 5-ALA-induced PpIX may be an appropriate treatment for patients with malignant brain tumors and should be assessed in clinical trials.

\section{Acknowledgements}

This study was supported by JSPS KAKENHI (grant no. 25462282).

\section{References}

1. Mimura S, Ito Y, Nagayo T, et al: Cooperative clinical trial of photodynamic therapy with photofrin II and excimer dye laser for early gastric cancer. Lasers Surg Med 19: 168-172, 1996.

2. Mlkvy P, Messmann H, Pauer M, et al: Distribution and photodynamic effects of meso-tetrahydroxyphenylchlorin (mTHPC) in the pancreas and adjacent tissues in the Syrian golden hamster. Br J Cancer 73: 1473-1479, 1996.

3. Yamamoto J, Hirano T, Li S, et al: Selective accumulation and strong photodynamic effects of a new photosensitizer, ATX-S10. $\mathrm{Na}$ (II), in experimental malignant glioma. Int $\mathrm{J}$ Oncol 27: 1207-1213, 2005.

4. Stummer W, Pichlmeier U, Meinel T, et al: Fluorescence-guided surgery with 5-aminolevulinic acid for resection of malignant glioma: a randomised controlled multicentre phase III trial. Lancet Oncol 7: 392-401, 2006.

5. Muller PJ and Wilson BC: Photodynamic therapy for malignant newly diagnosed supratentorial gliomas. J Clin Laser Med Surg 14: 263-270, 1996.

6. Yamamoto J, Yamamoto S, Hirano T, et al: Monitoring of singlet oxygen is useful for predicting the photodynamic effects in the treatment for experimental glioma. Clin Cancer Res 12: 7132-7139, 2006.

7. Schaffer M, Ertl-Wagner B, Schaffer PM, et al: Feasibility of photofrin II as a radiosensitizing agent in solid tumors - preliminary results. Onkologie 29: 514-519, 2006.

8. Luksiene Z, Juzenas P and Moan J: Radiosensitization of tumours by porphyrins. Cancer Lett 235: 40-47, 2006.

9. Berg K, Luksiene Z, Moan J and Ma L: Combined treatment of ionizing radiation and photosensitization by 5 -aminolevulinic acid-induced protoporphyrin IX. Radiat Res 142: 340-346, 1995.

10. Schaffer M, Schaffer PM, Jori G, et al: Radiation therapy combined with photofrin or 5-ALA: effect on Lewis sarcoma tumor lines implanted in mice. Preliminary results. Tumori 88: 407-410, 2002.

11. Takahashi J, Misawa M, Murakami M, et al: 5-Aminolevulinic acid enhances cancer radiotherapy in a mouse tumor model. Springerplus 2: 602, 2013.

12. Yamamoto J, Ogura S, Tanaka T, et al: Radiosensitizing effect of 5-aminolevulinic acid-induced protoporphyrin IX in glioma cells in vitro. Oncol Rep 27: 1748-1752, 2012.

13. Benda P, Someda K, Messer J and Sweet WH: Morphological and immunochemical studies of rat glial tumors and clonal strains propagated in culture. J Neurosurg 34: 310-323, 1971.

14. Schmidek HH, Nielsen SL, Schiller AL and Messer J: Morphological studies of rat brain tumors induced by $N$-nitrosomethylurea. J Neurosurg 34: 335-340, 1971.

15. Barth RF: Rat brain tumor models in experimental neuro-oncology: the 9L, C6, T9, F98, RG2 (D74), RT-2 and CNS-1 gliomas. J Neurooncol 36: 91-102, 1998.

16. Hagiya Y, Fukuhara H, Matsumoto K, et al: Expression levels of PEPT1 and ABCG2 play key roles in 5-aminolevulinic acid (ALA)-induced tumor-specific protoporphyrin IX (PpIX) accumulation in bladder cancer. Photodiagnosis Photodyn Ther 10: 288-295, 2013.

17. Ishizuka M, Hagiya Y, Mizokami Y, et al: Porphyrins in urine after administration of 5-aminolevulinic acid as a potential tumor marker. Photodiagnosis Photodyn Ther 8: 328-331, 2011.

18. Cerniglia GJ, Wilson DF, Pawlowski M, Vinogradov S and Biaglow J: Intravascular oxygen distribution in subcutaneous 9L tumors and radiation sensitivity. J Appl Physiol 82: 1939-1945, 1997.

19. Abels C, Heil P, Dellian M, et al: In vivo kinetics and spectra of 5-aminolaevulinic acid-induced fluorescence in an amelanotic melanoma of the hamster. Br J Cancer 70: 826-833, 1994. 
20. Abels C, Fritsch C, Bolsen K, et al: Photodynamic therapy with 5-aminolaevulinic acid-induced porphyrins of an amelanotic melanoma in vivo. J Photochem Photobiol B 40: 76-83, 1997.

21. Bozzini G, Colin P, Betrouni N, et al: Efficiency of 5-ALA mediated photodynamic therapy on hypoxic prostate cancer: a preclinical study on the Dunning R3327-AT2 rat tumor model. Photodiagnosis Photodyn Ther 10: 296-303, 2013.

22. Niclou SP, Danzeisen C, Eikesdal HP, et al: A novel eGFP-expressing immunodeficient mouse model to study tumor-host interactions. FASEB J 22: 3120-3128, 2008.

23. Prall F, Maletzki C and Linnebacher M: Microdensitometry of osteopontin as an immunohistochemical prognostic biomarker in colorectal carcinoma tissue microarrays: potential and limitation of the method in 'biomarker pathology'. Histopathology 61: 823-832, 2012

24. Kostron H, Swartz MR, Miller DC and Martuza RL: The interaction of hematoporphyrin derivative, light, and ionizing radiation in a rat glioma model. Cancer 57: 964-970, 1986.

25. Ito E, Yue S, Moriyama EH, et al: Uroporphyrinogen decarboxylase is a radiosensitizing target for head and neck cancer. Sci Transl Med 3: 67ra7, 2011

26. David S and Kroner A: Repertoire of microglial and macrophage responses after spinal cord injury. Nat Rev Neurosci 12 388-399, 2011

27. Lynch MA: The multifaceted profile of activated microglia. Mol Neurobiol 40: 139-156, 2009.

28. MacMicking J, Xie QW and Nathan C: Nitric oxide and macrophage function. Annu Rev Immunol 15: 323-350, 1997.
29. Boehm U, Klamp T, Groot M and Howard JC: Cellular responses to interferon-gamma. Annu Rev Immunol 15: 749-795, 1997.

30. Hussain SF, Yang D, Suki D, Grimm E and Heimberger AB: Innate immune functions of microglia isolated from human glioma patients. J Transl Med 4: 15, 2006.

31. Li W and Graeber MB: The molecular profile of microglia under the influence of glioma. Neuro Oncol 14: 958-978, 2012.

32. Filipazzi P, Huber V and Rivoltini L: Phenotype, function and clinical implications of myeloid-derived suppressor cells in cancer patients. Cancer Immunol Immunother 61: 255-263, 2012.

33. Skivka LM, Gorobets OB, Kutsenok VV, et al: 5-aminolevulinic acid mediated photodynamic therapy of Lewis lung carcinoma: a role of tumor infiltration with different cells of immune system. Exp Oncol 26: 312-315, 2004.

34. Ishizuka M, Abe F, Sano Y, et al: Novel development of 5-aminolevurinic acid (ALA) in cancer diagnoses and therapy. Int Immunopharmacol 11: 358-365, 2011.

35. Higashikawa F, Noda M, Awaya T, Tanaka T and Sugiyama M: 5-aminolevulinic acid, a precursor of heme, reduces both fasting and postprandial glucose levels in mildly hyperglycemic subjects. Nutrition 29: 1030-1036, 2013.

36. Starke RM, Williams BJ, Hiles C, et al: Gamma knife surgery for skull base meningiomas. J Neurosurg 116: 588-597, 2012.

37. Torres RC, Frighetto L, De Salles AA, et al: Radiosurgery and stereotactic radiotherapy for intracranial meningiomas. Neurosurg Focus 14: e5, 2003. 